\title{
APPROXIMATING EMBEDDINGS OF POLYHEDRA IN CODIMENSION THREE $\left({ }^{1}\right)$
}

BY

\section{J。L, BRYANT}

\begin{abstract}
Let $P$ be a $p$-dimensional polyhedron and let $Q$ be a PL $q$ manifold without boundary. (Neither is necessarily compact.) The purpose of this paper is to prove that, if $q-p \geq 3$, then any topological embedding of $P$ into $Q$ can be pointwise approximated by PL embeddings. The proof of this theorem uses the analogous result for embeddings of one PL manifold into another obtained by Cernavskii and Miller.
\end{abstract}

Introduction. Recently Černavskiı and Miller have shown that any topological embedding of a PL $m$-manifold $M$ into a PL $q$-manifold $Q$ can be approximated by PL embeddings provided $q-m \geq 3$. (See [5], [6], and [10].) In this paper we use this fact to prove that a topological embedding of an arbitrary $p$-dimensional polyhedron into $Q(q-p \geq 3)$ can be approximated by $\mathrm{PL}$ embeddings. This generalizes the results of Berkowitz [2] and Weber [13]. Our theorem can be stated as follows.

Theorem 1. Suppose that $P$ is a (not necessarily compact) p-dimensional polybedron, that $Q$ is a PL q-manifold without boundary $(q-p \geq 3)$, and that $f: P \rightarrow Q$ is a topological embedding. Then for each continuous function $\epsilon$ : $P \rightarrow(0, \infty)$ there exists a $P L$ embedding $g: P \rightarrow Q$ such that $d(f(x), g(x))<\epsilon(x)$ for each $x \in P$.

Moreover, if $R$ is a subpolybedron of $P$ on which $f \mid R$ is $P L$, then we may choose $g$ so that $g|R=f| R$.

The "moreover" part of Theorem 1 is simply an application of an isotopy theorem proved by Connelly [8], and we shall make no further reference to it.

Definitions and notations. By a polybedron we mean the underlying space of a locally finite simplicial complex embedded as a closed subset of some euclidean space (or manifold). We use the standard definition of piecewise linear (PL) map and PL manifold. When we say that $R$ is a subpolyhedron of a polyhedron $P$, we shall always assume that $R$ is a closed subset of $P$. If $f: P \rightarrow Q$ is a PL map,

Received by the editors November 18, 1970.

AMS 1970 subject classifications. Primary 57C05, 57C55, 57C35.

Key words and phrases. PL embedding, PL mapping, PL approximation, polyhedron, PL manifold.

(1) Partially supported by NSF Grants 11943 and 19964.

Copyright $\odot 1972$, American Mathematical Society 
then $S_{f}=\mathrm{Cl}\left\{x \mid f^{-1} f(x) \neq x\right\}$ shall denote the singular set of $f$. The unit ball in euclidean $n$-space $E^{n}$ is denoted by $B^{n} ; I=[0,1]$.

Given metric spaces $X$ and $Y$, a continuous function $\epsilon: X \rightarrow(0, \infty)$, and two mappings $f, g: X \rightarrow Y$, we define $d(f, g)<\epsilon$ to mean $d(f(x), g(x))<\epsilon(x)$ for each $x \in X$. (We ambiguously use $d$ to denote the metric of any space under consideration.)

If $K$ and $L$ are locally finite simplicial complexes, then $K \searrow L$ means that $K$ collapses to $L$ in the sense of [12]. In particular, we require that the collapse of $K$ to $L$ determine a proper deformation retraction of $|K|$ onto $|L|$ ("proper" meaning that inverse images of compact sets are compact). If $H$ is a subcomplex of $K$, then we use the notion of the trail of $H, \operatorname{tr} H$, under the collapse $K \succ L$ as defined by Zeeman in Chapter 7 of [16]. The important properties of tr $H$ are

(i) $\operatorname{dim} \operatorname{tr} H \leq 1+\operatorname{dim} H$,

(ii) $\operatorname{dim} L \cap \operatorname{tr} H \leq \operatorname{dim} H$, and

(iii) $K \searrow L \cup \operatorname{tr} H \searrow L$

as proved in Lemmas 44 and 45 of [16].

If $\epsilon:|K| \rightarrow(0, \infty)$ is continuous, then the collapse of $K$ to $L$ is called an $\epsilon$-collapse, written $K \epsilon^{\searrow} L$, if it gives rise to an $\epsilon$-deformation retraction of $|K|$ onto $|L|$. Observe that if $K \searrow L$ is an $\epsilon$-collapse and if $K^{(r)}$ is an rth derived subdivision of $K$, then the collapse of $K^{(r)}$ to $L^{(r)}$ can be arranged so that it is an $m \epsilon$-collapse, where $m$ depends only on the diameter of the simplexes of $K-L$ and the dimension of $K-L$.

A polyhedron $X$ collapses ( $\epsilon$-collapses) to a polyhedron $Y$ if there are locally finite simplicial complexes $K$ and $L$ such that $|K|=X,|L|=Y$, and $K$ collapses ( $\epsilon$-collapses) to $L$. Suppose that $X \succsim Y$ and $Z$ is a subpolyhedron of $X$. Let $K$ and $L$ be chosen as above so that $\left.K_{\epsilon}\right\rangle L$. Then there is an $r$ th derived subdivision $K^{(r)}$ containing a subcomplex $H$ such that $|H|=Z$ (see Chapter 1 , Lemma 4 of [16]). Define $\operatorname{tr} Z=|\operatorname{tr} H| \subset X$. Of course, tr $Z$ depends upon the triangulation involved. It follows, however, that in any event each of the collapses $X \searrow Y \cup \operatorname{tr} Z$ and $Y \cup \operatorname{tr} Z \searrow Y$ is an $m \epsilon$-collapse (for some $m$ as described above). This can be seen from our above remarks together $w$ ith the proof of Lemma 45 of [16].

Preliminary lemmas. The approximation theorem we require to prove Theorem 1 is stated as follows. (See [5], [6] and [10].)

Theorem 2. Suppose that $M$ and $Q$ are $P L$ manifolds of dimensions $m$ and $q$ respectively with $q-m \geq 3$, and that $f: M \rightarrow Q$ is a topological embedding. Then for each continuous $\epsilon: M \rightarrow(0, \infty)$ there exists a $P L$ embedding $g: M \rightarrow Q$ such that $d(f, g)<\epsilon$. 
I am deeply indebted to Robert Edwards, Richard Miller, and the referee for helping me simplify and correct many of the proofs appearing in the original version of this paper.

Lemma 1. Suppose that $f: B^{k} \rightarrow$ Int $Q(q-k \geq 3)$ is an embedding sucb that $f \mid$ Int $B^{k}$ is $P L$. Then there exists an extension of $f$ to an embedding $F$ : $B^{q} \rightarrow$ Int $Q$ such that $F \mid B^{q}-B d B^{k}$ in $P L$.

Proof. This is essentially a sequence of applications of Theorem 9 of [15].

The next lemma is a straightforward application of the Tietze extension theorem.

Lemma 2. Suppose that $f: B^{k} \rightarrow$ Int $Q$ is an embedding. Let $U$ be a neighborbood of $f\left(\right.$ Int $\left.B^{k}\right)$ in $Q$ and let $\epsilon:\left[Q-f\left(\operatorname{Bd} B^{k}\right)\right] \rightarrow(0, \infty)$ be continuous. Then there exist a neighborbood $V$ of $f\left(\right.$ Int $\left.B^{k}\right)$ in $Q$ and a continuous $\delta$ : Int $B^{k} \rightarrow$ $(0, \infty)$ such that, if $g: B^{k} \rightarrow V \cup f\left(B^{k}\right)$ is an embedding within $\delta$ of $f$ on Int $B^{k}$ that agrees with $f$ on $\mathrm{Bd} B^{k}$, then there exists an $\epsilon$-bomotopy $b_{t}: V \cup f\left(B^{k}\right) \rightarrow$ $U \cup f\left(B^{k}\right)$ such that $b_{0}=$ identity, $b_{t} \mid g\left(B^{k}\right)=$ identity for all $t \in I$, and $b_{1}$ is a retraction of $V \cup f\left(B^{k}\right)$ onto $g\left(B^{k}\right)$.

Lemma 3. Suppose that $f: B^{k} \rightarrow$ Int $Q, U$ and $\epsilon$ are as in Lemma 2. Then there exist a neighborbood $V$ of $f\left(\right.$ Int $\left.B^{k}\right)$ in $Q$ and continuous $\delta$ : Int $B^{k} \rightarrow$ $(0, \infty)$ and $\eta:\left[Q-f\left(\mathrm{Bd} B^{k}\right)\right] \rightarrow(0, \infty)$ such that, if $g: B^{k} \rightarrow V \cup f\left(B^{k}\right)$ is a $P L$ embedding within $\delta$ of $f$ on Int $B^{k}$ that agrees with $f$ on $\mathrm{Bd}^{k}$ and if $X \subset V$ is a polybedron that $\eta$ collapses to $g$ (Int $B^{k}$ ), then there exists an $\epsilon$-bomotopy $b_{t}$ : $V \cup f\left(\mathrm{Bd} B^{k}\right) \rightarrow U \cup f\left(\mathrm{Bd} B^{k}\right)$ such that $b_{0}=$ identity, $b_{t} \mid X=$ identity for all $t \in I$, and $b_{1}$ is a retraction of $V \cup f\left(B^{k}\right)$ onto $X \cup g\left(B^{k}\right)$.

Sketch of proof. Assume initially that $\delta$ and $V$ are chosen so as to correspond to $\epsilon / 2$ and $U$ as in Lemma 2. Suppose $X \subset V \eta$-collapses to $g$ (Int $B^{k}$ ), where $g: B^{k} \rightarrow V$ is PL on Int $B^{k}$ and within $\delta$ of $f$ on Int $B^{k}$. Let $N$ be a small, second-derived neighborhood of $X$ in $V$. Let $b^{\prime}$ be the 1-level of an $(\epsilon / 2)$-homotopy $b_{t}^{\prime}(t \in I)$ given by Lemma 2. Think of $N$ as the topological mapping cylinder of a map $\mathrm{Bd} N \rightarrow X$ given by the collapse $N \searrow X$, and let $a: \operatorname{Bd} N \times$ $I \rightarrow N$ be such that $\alpha(p, 0)=p, \alpha \mid B d N \times[0,1)$ is a homeomorphism onto $N-X$, $\alpha(\operatorname{Bd} N \times\{1\})=X, \alpha(p \times I)$ is small for $p \in \operatorname{Bd} N$ (say, less than $\eta(p)$ ). Let $\beta_{t}: X \rightarrow X(t \in I)$ be a strong $\eta$-deformation retraction of $X$ onto $g$ (Int $B^{k}$ ), with $\dot{\beta}_{0}=1$ and $\beta_{1}: X \rightarrow g\left(\right.$ Int $\left.B^{k}\right)$ a retraction. For $\delta$ and $\eta$ sufficiently small, the maps $b^{\prime} \mid \operatorname{Bd} N: \operatorname{Bd} N \rightarrow g\left(\right.$ Int $\left.B^{k}\right)$ and $\beta_{1} \alpha(-, 1): B d N \rightarrow g\left(\right.$ Int $\left.B^{k}\right)$ will be $(\epsilon / 2)$-homotopic via a homotopy $\gamma_{t}: B d N \rightarrow g\left(\right.$ Int $\left.B^{k}\right)(t \in I)$ with $\gamma_{0}(p)=$ $\beta_{1} \alpha(p, 1)$ and $\gamma_{1}(p)=b^{\prime}(p)$.

Define $b^{\prime \prime}: N \rightarrow X$ by 


$$
b^{\prime \prime}(\alpha(p, t))=\left\{\begin{array}{ll}
\gamma_{1-2 t}(\alpha(p, 0)), & 0 \leq t \leq 1 / 2 \\
\beta_{2-2 t}(\alpha(p, 1)), & 1 / 2 \leq t \leq 1
\end{array} \quad(p \in \operatorname{Bd} N),\right.
$$

and $b: V \rightarrow X$ by

$$
b(p)= \begin{cases}b_{1}^{\prime}(p), & p \in V-N \\ b^{\prime \prime}(p), & p \in N .\end{cases}
$$

Then $b$ is a retraction of $V$ onto $X$, and if $\delta$ and $\eta$ are sufficiently small, then $b$ will be the 1-level of the appropriate homotopy $b_{t}(t \in I)$ (extended to $f\left(\operatorname{Bd} B^{k}\right)$ via the identity, of course).

Lemma 4. Suppose that $f: B^{k} \rightarrow$ Int $Q$ is an embedding $(q-k \geq 4)$. Let $U$ be a neighborbood of $f\left(\right.$ Int $\left.B^{k}\right)$ in $Q$ and let $\epsilon:\left[Q-f\left(B d B^{k}\right)\right] \rightarrow(0, \infty)$ be continuous. Then there exist a neighborbood $V$ of $f\left(\right.$ Int $\left.B^{k}\right)$ in $Q$ and continuous functions $\delta$ : Int $B^{k} \rightarrow(0, \infty)$ and $\eta:\left[Q-f\left(\mathrm{Bd} B^{k}\right)\right] \rightarrow(0, \infty)$ satisfying the follow. ing conditions:

If $g: B^{k} \rightarrow V \cup f\left(B^{k}\right)$ is an embedding that is $P L$ and within $\delta$ of $f$ on Int $B^{k}$ and agrees with $f$ on $\mathrm{Bd} B^{k}$, if $Y$ is a polybedron in $V$ (embedded as a closed $P L$ subset of $\left.Q-f\left(\operatorname{Bd} B^{k}\right)\right)$ with $q-\operatorname{dim} Y \geq 4$, if $W$ is a neighborbood of $g$ (Int $B^{k}$ ) in $Q$, and if $X$ is a polybedron in $W$ sucb that $q-\operatorname{dim} X \geq 3$ and $X \searrow_{\eta} X \cap g\left(\right.$ Int $\left.B^{k}\right)$, then there exists an isotopy $b_{t}(t \in I)$ of $Q$ such that

(i) $b_{0}=$ identity,

(ii) $b_{t}=$ identity on $g\left(B^{k}\right) \cup X$ and outside of $U$,

(iii) $b_{1}(W) \supset Y$,

(iv) $d\left(b_{t}\right.$, identity $)<\epsilon$ on $Q-f\left(\mathrm{Bd}^{k}\right)$ for each $t \in I$.

This lemma is proved by using homotopies obtained from Lemma 2 together with standard engulfing arguments. The reader is referred particularly to [1], [11], and [14] for the engulfing techniques required here.

Lemma 5. Suppose that $f: B^{k} \rightarrow$ Int $Q$ is an embedding $(q-k \geq 4)$. Let $U$ be a neighborbood of $f\left(\operatorname{Int} B^{k}\right)$ in $Q$ and let $\epsilon:\left[Q-f\left(\mathrm{Bd} B^{k}\right)\right] \rightarrow(0, \infty)$ be continuous. Then there exist a neighborboad $V$ of $f\left(\right.$ Int $\left.B^{k}\right)$ and continuous functions $\eta:\left[Q-f\left(\mathrm{Bd} B^{k}\right)\right] \rightarrow(0, \infty)$ and $\delta:$ Int $B^{k} \rightarrow(0, \infty)$ satisfying the following conditions:

If $g: B^{k} \rightarrow V$ is a PL embedding within $\delta$ of $f$ that is $P L$ on Int $B^{k}$ and agrees with $f$ on $\mathrm{Bd} B^{k}$, if $X$ is a polybedron in $V(q-\operatorname{dim} X \geq 3)$ that collapses to $g$ (Int $\left.B^{k}\right)$ via an $\eta$-collapse, and if $Y$ is a polybedron in $V(q-\operatorname{dim} Y \geq 4)$, then there exists a polybedron $Z$ in $U$ sucb that 
(i) $Z \supset X \cup Y$,

(ii) $Z \underset{\epsilon}{\searrow} g\left(\right.$ Int $\left.B^{k}\right)$, and

(iii) $\operatorname{dim}(Z-X) \leq \operatorname{dim} Y+1$.

Proof. Suppose that $f: B^{k} \rightarrow$ Int $Q, U \supset f\left(\right.$ Int $\left.B^{k}\right)$, and $\epsilon:\left[Q-f\left(\mathrm{Bd} B^{k}\right)\right] \rightarrow$ $(0, \infty)$ are given as in the hypothesis of the lemma. Choose $V$ and $\delta$ corresponding to $U$ and $\epsilon / 3$ as in Lemma 3 , and let $g: B^{k} \rightarrow$ Int $Q$ be a $\delta$-approximation of $f$. Let $\eta=\epsilon / 3$. Suppose that $X$ and $Y$ satisfy the conditions in the lemma.

Let $W$ be a small, second-derived neighborhood of $X$ in $V$ such that every $r$ th derived subdivision of $W \eta$-collapses to $X$. From the conclusion of Lemma 3 we can obtain an isotopy $b_{t}(t \in I)$ that is fixed on $g\left(B^{k}\right) \cup X$ and outside $U$ such that $b_{1}(W) \supset Y$ and $d\left(b_{t}\right.$, identity $)<\epsilon / 3$ on $Q-f\left(B d B^{k}\right)$ for each $t \in I$. Let $Y_{1}=b_{1}^{-1}(Y)$, and let $Z_{1}=\operatorname{tr}\left(Y_{1} \cup X\right)$ under the collapse $W \succ X \succ$ $g\left(\right.$ Int $\left.B^{k}\right)$. Then $Z_{1}=X \cup \operatorname{tr} Y_{1}$ and $Z_{1}>g\left(\right.$ Int $\left.B^{k}\right)$ is a $2 \eta$-collapse. Let $Z=$ $b_{1}\left(Z_{1}\right)=X \cup b_{1}\left(\operatorname{tr} Y_{1}\right)$ (since $b_{1} \mid X=$ identity). Then $Z Z_{\epsilon} g\left(\right.$ Int $\left.B^{k}\right)$ and $\operatorname{dim}(Z-X) \leq \operatorname{dim} Y+1$.

Main lemmas. Suppose that $J$ is a complex and that $\sigma$ is a simplex of $J$. Let $K=\operatorname{St}(\sigma ; J), L=\operatorname{Bd} \sigma * \operatorname{LK}(\sigma ; J), \stackrel{\circ}{K}=|K|-|L|$, and $\stackrel{\circ}{\sigma}=$ Int $\sigma$. Denote by $\hat{\sigma}$ the barycenter of $\sigma$. There is a triangulation $K_{1}$ of $|K|-\mathrm{Bd} \sigma$ such that $K_{1} \succ H_{1}$, where $H_{1} \subset K_{1}$ and $\left|H_{1}\right|=\stackrel{\circ}{\sigma}$. Thus we have $\operatorname{tr} Z$ defined for a subpolyhedron $Z$ of $\left|K_{1}\right|$. Set $k=\operatorname{dim} K$ and assume $q-k \geq 3$ throughtout.

Given $J, \sigma, K$, and $L$ as above, let us consider $|K|$ as the cone $\hat{\sigma} *|L|$. If $x \in|K|$, let $[\hat{\sigma}, x]$ denote the segment joining $\hat{\sigma}$ and $x$ in $|K|$. Similarly, let us consider $B^{q}$ as the cone $0 * \mathrm{Bd} B^{q}$, with subcone $0 * \mathrm{Bd} B^{j}$ (where $j=\operatorname{dim} \sigma$ ), and let $[0, y]$ denote the segment joining $y \in B^{q}$ to 0 in this cone structure. Let $g: \sigma \rightarrow B^{j}$ be a fixed PL homeomorphism with $g(\hat{\sigma})=0$. We use this notation in the following lemmas.

Lemma 6. Suppose that $f: \sigma \rightarrow Q$ is an embedding and $\epsilon: \stackrel{\circ}{\sigma} \rightarrow(0, \infty)$ is continuous. Then there exists a continuous $\delta: \stackrel{\circ}{\sigma} \rightarrow(0, \infty)$ such that $x, y \in \stackrel{\circ}{\sigma}$ and $d(f(x), f(y))<\delta(x)$ imply $d(f(z), f(\theta(z)))<\epsilon(z)$ for each $z \in[\hat{\sigma}, x]$, where $\theta$ : $[\hat{\sigma}, x] \rightarrow[\hat{\sigma}, y]$ is the linear bomeomorphism satisfying $\theta(\hat{\sigma})=\hat{\sigma}$ and $\theta(x)=y$.

The proof of Lemma 6 is obtained by applying the continuity of $f$ and $f^{-1}$ and shall be left for the reader. The next two lemmas are successive generalizations of Lemma 6.

Lemma 7. Given $f: \sigma \rightarrow Q$ and $\epsilon: \stackrel{\circ}{\sigma} \rightarrow(0, \infty)$ as in Lemma 6 , there exist $\delta, \eta: \stackrel{\circ}{\sigma} \rightarrow(0, \infty)$ such that if $g_{0}, g_{1}: \sigma \rightarrow Q$ are mappings with $d\left(g_{j}(x), f(x)\right)<$ $\eta(x)$ for each $x \in \stackrel{\circ}{\sigma}$, then $x, y \in \stackrel{\circ}{\sigma}$ and $d\left(g_{0}(x), g_{1}(y)\right)<\delta(x)$ imply $d\left(g_{0}(z), g_{1}(\theta(z))\right)<\epsilon(z)$ for each $z \in[\hat{\sigma}, x]$. 
Lemma 8. Suppose that $A$ and $B$ are cones over $\hat{\sigma}$ each containing $\sigma$ as $a$ subcone, that $f: \sigma \rightarrow Q$ is an embedding, and that $\epsilon:[A-\operatorname{Bd} \sigma] \rightarrow(0, \infty)$ is continuous. Then there exist $\delta:[A-\operatorname{Bd} \sigma] \rightarrow(0, \infty)$ and $\eta: \stackrel{\circ}{\sigma} \rightarrow(0, \infty)$ satisfying the following properties:

If $G_{0}: A \rightarrow Q$ and $G_{1}: B \rightarrow Q$ are mappings with $d\left(\left.G_{i}\right|^{\circ}, f \mid{ }^{\circ}\right)<\eta(i=0,1)$, then there exist neighborboods $U$ of $\stackrel{\circ}{\sigma}$ in $A$ and $V$ of $\stackrel{\circ}{\sigma}$ in $B$ such that $x \in U$, $y \in V$, and $d\left(G_{0}(x), G_{1}(y)\right)<\delta(x)$ imply $d\left(G_{0}(z), G_{1}(\theta(z))\right)<\epsilon(z)$ for each $z \epsilon$ $[\hat{\sigma}, x]$.

Lemma 9. Suppose $f: \stackrel{\circ}{K} \rightarrow Q$ is a closed embedding with $f \mid \stackrel{K}{-}-\stackrel{\circ}{\sigma} P L$. Then for each $\epsilon: \stackrel{\circ}{K} \rightarrow(0, \infty)$ and each neighborbood $N$ of $\stackrel{\circ}{\sigma}$ in $\stackrel{\circ}{K}$, there exists a $P L$ map $G: \stackrel{\circ}{K} \rightarrow Q$ such that

(9.1) $G|\stackrel{\circ}{K}-N=f| \stackrel{\circ}{K}-N$,

(9.2) $\left.G\right|_{\sigma} ^{\circ}$ is a $P L$ embedding,

(9.3) $d(f, G)<\epsilon$,

(9.4) $S_{G} \subset N$, and

(9.5) $G$ is in general position.

Proof. This is a straightforward application of Theorem 2 (as applied to the embedding $f \mid \stackrel{\circ}{\sigma}: \stackrel{\circ}{\sigma} \rightarrow Q)$ and general position.

Lemma 10. Suppose that $f: \stackrel{\circ}{K} \rightarrow Q$ is a closed embedding with $f \mid \stackrel{\circ}{K}-\stackrel{\circ}{\sigma} P L$. Given $\epsilon: Q \rightarrow(0, \infty)$ there exist a neighborbood $N$ of $\stackrel{\circ}{\sigma}$ in $\stackrel{\circ}{K}$ and continuous functions $\eta: Q \rightarrow(0, \infty)$ and $\delta: \stackrel{\circ}{K} \rightarrow(0, \infty)$ satisfying the following conditions:

If $G: K \rightarrow Q$ is a $P L$ map satisfying (9.1)-(9.5) of Lemma 9 with $\{\delta, N\}$ replacing $\{\epsilon, N\}$ and if $X_{1}$ and $Z_{1}$ are polybedra such that

(10.1) $S_{G} \cup \stackrel{\circ}{\sigma} \subset X_{1}=\operatorname{tr} X_{1} \subset N$,

(10.2) $Y_{1}=G\left(X_{1}\right) \subset Z_{1} \eta \searrow G(\stackrel{\circ}{\sigma})$, and

(10.3) $\operatorname{dim}\left[\left(Z_{1}-Y_{1}\right) \cap G(\stackrel{\circ}{K})\right] \leq k-j(j \geq 4)$,

then there exist polybedra $X_{2}$ and $Z_{2}$ sucb that

(10.4) $X_{1} \subset X_{2}=\operatorname{tr} X_{2} \subset \stackrel{\circ}{\circ}$,

(10.5) $Y_{2}=G\left(X_{2}\right) \subset Z_{2} \epsilon_{0} G(\stackrel{\circ}{\sigma})$, and

(10.6) $\operatorname{dim}\left[\left(Z_{2}-Y_{2}\right) \cap G(K)\right] \leq k-j-1$.

Proof. Given $\epsilon: Q \rightarrow(0, \infty)$, let $U=N_{\epsilon}(f(\stackrel{\circ}{\sigma}))$, and choose $\delta$, $\eta$, and $V$ as in Lemma 5. Choose $N$ to be a regular neighborhood of $\stackrel{\circ}{\sigma}$ in $\stackrel{\circ}{K}$ such that $N=\operatorname{tr} N$ and $f(N) \subset V$. Assume that $G: \stackrel{\circ}{K} \rightarrow Q$ satisfies (9.1)-(9.5) of Lemma 9 with $\delta$ replacing the $\epsilon$ of Lemma 9 and that $G(N) \subset V$.

Suppose that $X_{1} \subset N$ and $Z_{1} \subset Q$ satisfy (10.1)-(10.3). Let $X_{2}=$ $\operatorname{tr}\left(G^{-1}\left(Z_{1}\right)\right)$, and let $Y_{2}=G\left(X_{2}\right)$. Then $\operatorname{dim}\left(X_{2}-X_{1}\right)=\operatorname{dim}\left(Y_{2}-Y_{1}\right) \leq k-j+1$ 
$(\leq q-6)$. Observe that if $\delta$ and $\eta$ are sufficiently small, then $G^{-1}\left(Z_{1}\right) \subset N$, and so $Y_{2} \subset V$. Now apply Lemma 5 with $Z_{1}$ replacing $X$ and $\mathrm{Cl}\left(Y_{2}-Y_{1}\right)$ replacing $Y$. This gives us a polyhedron $Z_{2} \subset Q$ satisfying

(i) $Z_{1} \cup \mathrm{Cl}\left(Y_{2}-Y_{1}\right) \subset Z_{2}$,

(ii) $Z_{2 \epsilon} \searrow G(\stackrel{\circ}{\sigma})$, and

(iii) $\operatorname{dim}\left(Z_{2}-Z_{1}\right) \leq \operatorname{dim}\left(Y_{2}-Y_{1}\right)+1 \leq k-j+2$.

Putting $Z_{2}$ in general position with respect to $G(K)$ (keeping $Z_{1} \cup Y_{2}$ fixed), we have (since $Z_{1} \cap G(\stackrel{\circ}{K}) \subset Y_{2}$ )

$$
\begin{aligned}
\operatorname{dim}\left[\left(Z_{2}-Y_{2}\right) \cap G(\stackrel{\circ}{K})\right] & \leq \operatorname{dim}\left[\left(Z_{2}-Z_{1}\right) \cap G(\stackrel{\circ}{K})\right] \\
& \leq(k-j+2)+k-q \leq k-j-1 .
\end{aligned}
$$

Lemma 11. Suppose that $f: \stackrel{\circ}{K} \rightarrow Q$ is a closed embedding with $f \mid \stackrel{\circ}{K}-\stackrel{\circ}{\sigma} P L$. Given $\epsilon: Q \rightarrow(0, \infty)$ and a neighborbood $N$ of $\stackrel{\circ}{\sigma}$ in $\stackrel{\circ}{K}$, there exists $\delta: \stackrel{\circ}{K} \rightarrow(0, \infty)$ such that, if $G: \stackrel{\circ}{K} \rightarrow Q$ is a PL map satisfying (9.1)-(9.5) of Lemma 9 with $\{\delta, N\}$ replacing $\{\epsilon, N\}$, then there exist polybedra $X \subset N$ and $Z \subset Q$ satisfying

(11.1) $\stackrel{\circ}{\sigma} \cup S_{G} \subset X=\operatorname{tr} X \subset N$,

(11.2) $G(X)=Z \cap G(\stackrel{\circ}{)}$, and

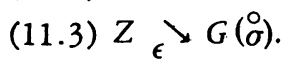

Proof. Apply Lemma 10 inductively.

The next lemma is the key lemma of this paper. Its proof depends upon a result of J. Cobb announced in [7]. The specific form of Cobb's theorem we wish to consider is the following.

Theorem 3 (Cobb [7]). Suppose that $L$ is a subcomplex of a finite k-dimensional complex $K$ and that $f$ is an embedding of $|K|$ into a PL q-manifold $Q(q-k \geq 3)$ such that $f|| L \mid$ and $f|| K|-| L \mid$ are $P L$. Then for each $\epsilon>0$ there exists an isotopy $b: Q \times I \rightarrow Q \times I$ sucb that

(i) $b_{0}=$ identity,

(ii) $b_{1} f:|K| \rightarrow Q$ is $P L$,

(iii) $b_{t} f|| L|=f||L|$, and

(iv) $b \mid(Q \times I)-(f(|L|) \times\{0\})$ is $P L$.

Lemma 12. Suppose that $f:|K| \rightarrow Q$ is an embedding with $f \mid K-\stackrel{\circ}{\sigma} P L$. Then for each $\epsilon: \stackrel{\circ}{K} \rightarrow(0, \infty)$ and each neighborbood $N$ of $\stackrel{\circ}{\sigma}$ in $\stackrel{\circ}{K}$, there exists an embedding $f^{\prime}:|K| \rightarrow Q$ such that

(i) $f^{\prime}|| L|\cup(|K|-N)=f||L| \cup(|K|-N)$,

(ii) $f^{\prime} \mid K$ is $P L$, and

(iii) $d\left(f(x), f^{\prime}(x)\right)<\epsilon(x)$ for $x \in \stackrel{\circ}{K}$.

Proof. Given $\zeta: \stackrel{\circ}{K} \rightarrow(0, \infty)$, choose $\delta: \stackrel{\circ}{K} \rightarrow(0, \infty)$ and $\eta: \stackrel{\circ}{\sigma} \rightarrow(0, \infty)$ as in 
Lemma 8 corresponding to $\epsilon=\zeta$. Let $G: \stackrel{\circ}{K} \rightarrow(Q-f(L))$ be a PL map obtained from Lemma 11 with $d(f, G)<\eta$ on $\stackrel{\circ}{\sigma}$ and let $X$ and $Z$ be the associated polyhedra having the property that $\stackrel{\circ}{\sigma} \cup S_{G} \subset X=\operatorname{tr} X \subset N, G(X)=Z \cap G(K)$, and $Z \searrow_{\zeta} G(\stackrel{\circ}{\sigma})$. Triangulate $\stackrel{\circ}{K}$ and $Q-f(|L|)$ so that $G: \stackrel{\circ}{K} \rightarrow Q-f(|L|)$ is simplicial, and let $U^{\prime}$ and $V^{\prime}$ be small, second-derived neighborhoods of $X$ in $\stackrel{\circ}{K}$ and $Z$ in $Q-f(|L|)$, respectively, such that $G\left(U^{\prime}\right)=V^{\prime} \cap G(\stackrel{\circ}{K})$.

Assume that $\delta$ is chosen so that $G$ extends to a map of $|K|$ into $Q$ (which we shall still call $G$ ) that agrees with $f$ on $|L|$.

Let $H: B^{q} \rightarrow Q$ be an embedding such that

$H \mid\left(B^{q}-B d B^{j}\right)$ is PL $(j=\operatorname{dim} \sigma)$,

$H(0)=G(\hat{\sigma})$, and

$H g=G$.

Let $U$ be the closure (in $|K|$ ) of a small, second-derived neighborhood of $\stackrel{\circ}{\sigma}$ in $K$ lying in the closure of $N$ in $|K|$, and let $V$ be the closure in $B^{q}$ of a small, second-derived neighborhood of Int $B^{j}$ in $B^{q}-B d B^{j}$, both chosen so as to satisfy the conclusion of Lemma 8 with $\zeta$ replacing $\epsilon$. We shall make the further assumption that $U$ is starlike in $K$ from $\hat{\sigma}, V$ is starlike in $B^{q}$ from 0 , and $H(V) \cap G(|K|) \subset G(N \cup \sigma)$.

Since $X=\operatorname{tr} X \succ \stackrel{\circ}{\sigma}$, there exists a small homeomorphism $F_{1}:|K| \rightarrow|K|$ such that

$F_{1} \mid \stackrel{\circ}{K}$ is $\mathrm{PL}$,

$F_{1}|| L \mid \cup(|K|-N) \cup \sigma=$ identity, and

$F_{1}(U) \cap \stackrel{\circ}{K}=U^{\prime}$.

If $\eta$ is sufficiently small and if $X$ is suitably chosen, then we can guarantee that $d\left(G(x), G F_{1}(x)\right)<\delta(x) / 2$ for $x \in \stackrel{\circ}{K}$.

Similarly, there exists a small homeomorphism $F_{2}: Q \rightarrow Q$ such that

$F_{2} \mid f(|L|) \cup G(\sigma)=$ identity,

$F_{2} \mid Q-f(|L|)$ is $\mathrm{PL}$,

$F_{2} H(V)-f(|L|)=V^{\prime}$, and

$d\left(F_{2} G(x), G(x)\right)<\zeta(x) / 3$ for $x \in \stackrel{\circ}{K}$.

Thus, $G F_{1}(\mathrm{Bd} U)=G(|K|) \cap F_{2} H(\mathrm{Bd} V)$ is homeomorphic to $|L|$. Let $L^{\prime}=$ $H^{-1} F_{2}^{-1}\left(G(|K|) \cap F_{2} H(B d V)\right) \subset B d V$, and let $K^{\prime}=L^{\prime} * 0 \subset V \subset B^{q}$. Observe that $U=\hat{\sigma} * \mathrm{Bd} U$ and $G F_{1}(\mathrm{Bd} U)=F_{2} H\left(L^{\prime}\right)$.

Define $F_{3}: U \rightarrow V$ by $F_{3}(x)=H^{-1} F_{2}^{-1} G F_{1}(x)$ if $x \in \operatorname{Bd} U$ and $F_{3} \mid[\hat{\sigma}, x]=$ $\theta:[\hat{\sigma}, x] \rightarrow\left[0, F_{3}(x)\right]$ is the linear homeomorphism described previously. Now define $G^{\prime}:|K| \rightarrow Q$ by

$$
\begin{array}{ll}
G^{\prime}(x)=F_{2} H F_{3}(x) & \text { if } x \in U, \\
G^{\prime}(x)=G F_{1}(x) & \text { if } x \notin U .
\end{array}
$$


Then, assuming $\eta<\zeta, G^{\prime}$ is an embedding that closely approximates $f$.

Let $H_{1}: V \rightarrow B^{q}$ be a homeomorphism such that $H_{1} \mid B^{j}=$ identity and $H_{1} \mid B^{q}-\mathrm{Bd} B^{j}$ is PL. Then $G_{1}: U \rightarrow B^{q}$, defined by $G_{1}=H_{1} H^{-1} F_{2}^{-1} G^{\prime}$, is a proper embedding of the cone $\hat{\sigma} * \mathrm{Bd} U$ into $B^{q}=0 * \mathrm{Bd} B^{q}$ such that $G{ }_{1} \mid \sigma$ is $\mathrm{PL}$ and $G_{1} \mid U-\sigma$ is $\mathrm{PL}$.

Consider $G_{1}\left|\mathrm{Bd} U: \mathrm{Bd} U \rightarrow \mathrm{Bd} B^{q} . G_{1}\right| \mathrm{Bd} B^{j}$ is $\mathrm{PL}$ and $G_{1} \mid \mathrm{Bd} U-\mathrm{Bd} B^{j}$ is PL. Hence, by Theorem 3, there exists a small isotopy $k_{t}(t \in I)$ of $\mathrm{Bd} B^{q}$ such that $k_{0}=$ identity, $k_{1} G_{1}(\mathrm{Bd} U)$ is a subpolyhedron of $\mathrm{Bd} B^{q}, k_{1} G_{1} \mid \mathrm{Bd} \sigma=$ $G_{1} \mid \mathrm{Bd} \sigma$, and $k \mid\left(\mathrm{Bd} B^{q} \times I\right)-\left(\mathrm{Bd} B^{j} \times\{0\}\right)$ is $\mathrm{PL}$. Copy this isotopy in a small $\mathrm{PL}$ collar $W$ of $\mathrm{Bd} B^{q}$ in $B^{q}$. Let $U_{1}$ be $U$ minus a small collar of $\mathrm{Bd} U$ in $U$ and obtain a PL embedding $G_{2}: U_{1} \rightarrow \overline{B^{q}-W}$ by coning over $k_{1} G_{1}(\operatorname{Bd} U) \subset \mathrm{Bd} W$.

Extend $G_{2}: U_{1} \rightarrow \overline{B^{q}-W}$ to $G_{3}: U \rightarrow B^{q}$ by sending $\overline{U-U_{1}}$ to $k(\mathrm{Bd} U \times I) \subset \stackrel{W}{W}$ in a natural way. Then $G_{3}: U \rightarrow B^{q}$ is a proper embedding, $G_{3} \mid U-\mathrm{Bd} \sigma$ is $\mathrm{PL}, G_{3}\left|\mathrm{Bd} U=G_{1}\right| \mathrm{Bd} U$, and $G_{3}$ is a closed approximation to $G_{1}$. Thus if $G_{3}$ is sufficiently close to $G_{1}$, then the embedding $f^{\prime}:|K| \rightarrow Q$ defined by

$$
\begin{array}{ll}
f^{\prime}(x)=F_{2} H H_{1}^{-1} G_{3}(x) & \text { if } x \in U, \\
f^{\prime}(x)=G^{\prime}(x) & \text { if } x \notin U
\end{array}
$$

is $\mathrm{PL}$ on $\stackrel{\circ}{K}$, is an approximation of $f$ on $\stackrel{\circ}{K}$, and agrees with $f$ on $|L| \cup(|K|-N)$.

Proof of Theorem 1. Suppose that $f: P \rightarrow Q$ is a topological embedding. Let $J$ be a triangulation of $P$. Apply Theorem 2 to the open $p$-dimensional simplexes of $J$ to obtain an embedding $f_{1}: P \rightarrow Q$ that approximates $f$ and satisfies the two properties: $f_{1}|| J^{p-1}|=f|\left|J^{p-1}\right|$ and $f_{1} \mid\left(|J|-\left|J^{p-1}\right|\right)$ is PL. Now proceed inductively using Lemma 12.

Appendix. Observe that in the proof of Lemma 12 we applied Theorem 3 in dimension $q-1$ to a compact polyhedron to get Lemma 12 in dimension $q$. In view of the fact that a proof of Cobb's theorem [7] (Theorem 3) has not appeared as yet, we shall outline a proof of Theorem 3 in dimension $q$, assuming Theorem 1 in dimension $q$. (The idea appears to be essentially the same as that indicated in [71.) We shall require an isotopy theorem and an engulfing the orem due to Edwards [9] and Bryant-Seebeck [3], respectively.

Isotopy Theorem (Edwards [9]). Suppose that $L$ is a subcomplex of a finite $k$-dimensional complex $K$ and that $f:|K| \rightarrow Q(q-k \geq 3)$ is a topological embedding such that $f|| L \mid$ is $P L$. Then, given $\epsilon>0$, there exists $\delta>0$ such that if $g_{i}:|K| \rightarrow Q(i=1,2)$ is a $P L$ embedding sucb that $g_{i}|| L|=f||L|$ and $d\left(g_{i}, f\right)<\delta$, then $g_{1}$ and $g_{2}$ are $P L \epsilon$-ambient isotopic in $Q$ via an isotopy that is fixed outside $N_{\epsilon}(f(|K|-|L|))$. 
Engulfing Theorem (Bryant-Seebeck [3]). Suppose that $X$ is a compact $k$ dimensional $A N R$ in $Q(q-k \geq 3, q \geq 5)$ such that $Q-X$ is $1-U L C$ (uniformly locally simply connected) and $A \subset X$ is closed in $X$. Then, given $\epsilon>0$, there exists $\delta>0$ such that if $g: X \rightarrow Q$ is an embedding with $d(g, 1)<\delta$ and $g \mid A=1$ and if $U$ is an open set in $Q$ containing $g(X)$, then there exists a $P L \epsilon$-isotopy $b_{t}(t \in I)$ of $Q$ that is fixed outside $N_{\epsilon}(X-A)$ sucb that $b_{0}=1$ and $b_{1}(U) \supset X$. Moreover, if $X$ is a polybedron and $g: X \rightarrow Q$ is $P L$ then $b_{t}(t \in I)$ can be chosen so as to fix $g(X)$.

(This statement is slightly stronger than the statement of Theorem 2.1 of [3], but its proof is contained implicitly therein. Indeed, it has been pointed out to the author that the above refinement of Theorem 2.1 of [3] is actually needed to prove some of the theorems of [3]. The "moreover" part was obtained by Černavskiř in [5].)

Proof of Theorem 3 in dimension $q$, assuming Theorem 1 in dimension $q$. Suppose that $L$ is a subcomplex of a finite $k$-complex $K$ and that $f:|K| \rightarrow Q$ $(q-k \geq 3)$ is an embedding such that $f|| L \mid$ is PL and $f|| K|-| L \mid$ is PL. (We shall assume that $q \geq 5$, since both Theorem 1 and Theorem 3 are well known in dimension $q=4$ [4].) Let $N_{1}, N_{2}, \cdots$ be a sequence of PL neighborhoods of $|L|$ in $|K|$ such that $N_{i+1} \subset \operatorname{Int} N_{i}$ and $\bigcap_{i=1}^{\infty} N_{i}=|L|$, and let $P_{i}=$ $|L| \cup \mathrm{Cl}\left(|K|-N_{i}\right), i=1,2, \cdots$.

Now proceed just as in the proof of Theorem 2 of [3]. Start with a PL approximation $f^{\prime}:|K| \rightarrow Q$ of $f$ that agrees with $f$ on $P_{1}$. Use the Isotopy Theorem and Engulfing Theorem to construct sequences of small PL pushes $\left\{G_{i}\right\}_{i=1}^{\infty}$ and $\left\{G_{i}^{\prime}\right\}_{i=1}^{\infty}$ of $(Q, f(|K|))$ satisfying

(1) $G=\lim _{i \rightarrow \infty} G_{i} \cdots G_{1}$ and $G^{\prime}=\lim _{i \rightarrow \infty} G_{i}^{\prime} \cdots G_{1}^{\prime}$ are small pushes of $Q$,

(2) $G f=G^{\prime} f^{\prime}$,

(3) $G_{i} \cdots G_{1} f\left|P_{i}=G_{i}^{\prime} \cdots G_{1}^{\prime} f^{\prime}\right| P_{i}$,

(4) the PL isotopy of $G_{i+1}$ (respectively, $G_{i+1}^{\prime}$ ) to the identity is fixed on $G_{i} \cdots G_{1} f\left(|K|-N_{i}\right.$ ) (respectively, $G_{i}^{\prime} \cdots G_{1}^{\prime} f^{\prime}\left(|K|-N_{i}\right)$ ). The homeomorphism $G^{\prime} G^{-1}$ is isotopic to the identity via the appropriate isotopy of $Q$.

\section{REFERENCES}

1. R. H. Bing, Radial engulfing, Conference on the Topology of Manifolds (Michigan State Univ., E. Lansing, Mich., 1967), Prindle, Weber and Schmidt, Boston, Mass., 1968, pp. 1-18. MR $38 \# 6560$.

2. H. W. Berkowitz, Piecewise linear approximations of homeomorphisms (preprint). 
3. J. L. Bryant and C. L. Seebeck III, Locally nice embeddings of polyhedra, Quart. J. Math. Oxford Ser. (2) 19 (1968), 257-274. MR 38 \#2751.

4. J. C. Cantrell, n-frames in euclidean k-space, Proc. Amer. Math. Soc. 15 (1964), 574-578. MR $29 \# 1627$.

5. A. V. Černavskiř, Topological embeddings of manifolds, Dokl. Akad. Nauk SSSR 187 (1969), 1247-1250 = Soviet Math. Dokl. 10 (1969), 1037-1040. MR 41 \#4547.

6. - Piecewise linear approximations of embeddings of cells and spheres in codimensions higher than two, Mat. Sb. 80 (122) (1969), 339-364= Math. USSR Sb. 9 (1969), 321-344. MR 40 \#4957.

7. J. I. Cobb, Taming almost PL embeddings, Notices Amer. Math. Soc. 15 (1968), 371. Abstract \#68T-241.

8. R. Connelly, Unknotting close embeddings of polyhedra in codimension greater than two, Ph. D. Dissertation, University of Michigan, Ann Arbor, Mich., 1969.

9. R. D. Edwards, The equivalence of close piecewise-linear embeddings (to appear).

10. R. J. Miller, Approximating codimension three embeddings, Ann. of Math. (to appear).

11. C. L. Seebeck III, Collaring an (n-1)-manifold in an n-manifold, Trans. Amer. Math. Soc. 148 (1970), 63-68. MR $41 \# 2692$.

12. A. Scott, Infinite regular neighbourhoods, J. London Math. Soc. 42 (1967), 245253. MR $35 \# 3672$.

13. C. Weber, Plongements de polyèdres dans le domaine métastable, Comment Math. Helv. 42 (1967), 1-27. MR 38 \#6606. 298.

14. P. Wright, Radial engulfing in codimension three, Duke Math. J. 38 (1971), 295-

15. E. C. Zeeman, Unknotting combinatorial balls, Ann. of Math. (2) 78 (1963), 501526. MR $28 \# 3432$.

16. - Seminar on combinatorial topology, Inst. Hautes Études Sci., Paris, 1963 (mimeograph notes).

DEPARTMENT OF MATHEMATICS, FLORIDA STATE UNIVERSITY, TALLAHASSEE, FLORIDA 32306 